\title{
Following the Birth of a Nanoplasma Produced by an Ultrashort Hard-X-Ray Laser in Xenon Clusters
}

\author{
Yoshiaki Kumagai, ${ }^{1}$ Hironobu Fukuzawa, ${ }^{1,2}$ Koji Motomura, ${ }^{1}$ Denys Iablonskyi, ${ }^{1}$ Kiyonobu Nagaya, ${ }^{2,3}$ Shin-ichi Wada, ${ }^{2,4}$ \\ Yuta Ito, ${ }_{1}$ Tsukasa Takanashi, ${ }^{1}$ Yuta Sakakibara, ${ }^{1}$ Daehyun You, ${ }^{1}$ Toshiyuki Nishiyama, ${ }^{3}$ Kazuki Asa, ${ }^{3}$ Yuhiro Sato, ${ }^{3}$ \\ Takayuki Umemoto, ${ }^{4}$ Kango Kariyazono, ${ }^{4}$ Edwin Kukk, ${ }^{5}$ Kuno Kooser, ${ }^{5,6}$ Christophe Nicolas, ${ }^{7}$ Catalin Miron, ${ }^{7,8,9}$ \\ Theodor Asavei, ${ }^{8}$ Liviu Neagu, ${ }^{8}$ Markus S. Schöffler, ${ }^{10}$ Gregor Kastirke, ${ }^{10}$ Xiao-jing Liu, ${ }^{11}$ Shigeki Owada, ${ }^{2}$ \\ Tetsuo Katayama, ${ }^{12}$ Tadashi Togashi, ${ }^{12}$ Kensuke Tono, ${ }^{12}$ Makina Yabashi, ${ }^{2}$ Nikolay V. Golubev, ${ }^{13}$ \\ Kirill Gokhberg, ${ }^{13}$ Lorenz S. Cederbaum, ${ }^{13}$ Alexander I. Kuleff, ${ }^{13}$ and Kiyoshi Ueda ${ }^{1,2, *}$ \\ ${ }^{1}$ Institute of Multidisciplinary Research for Advanced Materials, Tohoku University, Sendai 980-8577, Japan \\ ${ }^{2}$ RIKEN SPring-8 Center, Sayo, Hyogo 679-5148, Japan \\ ${ }^{3}$ Department of Physics, Kyoto University, Kyoto 606-8502, Japan \\ ${ }^{4}$ Department of Physical Science, Hiroshima University, Higashi-Hiroshima 739-8526, Japan \\ ${ }^{5}$ Department of Physics and Astronomy, University of Turku 20014 Turku, Finland \\ ${ }^{6}$ Institute of Physics, University of Tartu, Wilhelm Ostwaldi Str 1, 50411 Tartu, Estonia \\ ${ }^{7}$ Synchrotron SOLEIL, L'Orme des Merisiers, Saint-Aubin, BP 48, FR-91192 Gif-sur-Yvette Cedex, France \\ ${ }^{8}$ Extreme Light Infrastructure-Nuclear Physiscs (ELI-NP), "Horia Hulubei” National Institute for Physics \\ and Nuclear Engineering, 30 Reactorului Street, RO-077125 Măgurele, Judeţul Ilfov, Romania \\ ${ }^{9}$ LIDYL, CEA, CNRS, Université Paris-Saclay, CEA Saclay, 91191 Gif-sur-Yvette, France \\ ${ }^{10}$ Institut für Kernphysik, Goethe-Universität Frankfurt, 60438 Frankfurt am Main, Germany \\ ${ }^{11}$ School of Physics and Nuclear Energy Engineering, Beihang University, \\ Beijing 100191, People's Republic of China \\ ${ }^{12}$ Japan Synchrotron Radiation Research Institute (JASRI), Sayo, Hyogo 679-5198, Japan \\ ${ }^{13}$ Theoretische Chemie, PCI, Universität Heidelberg, 69120 Heidelberg, Germany
}

(Received 6 October 2017; revised manuscript received 4 June 2018; published 2 August 2018)

X-ray free-electron lasers (XFELs) made available a new regime of x-ray intensities, revolutionizing the ultrafast structure determination and laying the foundations of the novel field of nonlinear x-ray optics. Although earlier studies revealed nanoplasma formation when an XFEL pulse interacts with any nanometer-scale matter, the formation process itself has never been decrypted and its timescale was unknown. Here we show that time-resolved ion yield measurements combined with a near-infrared laser probe reveal a surprisingly ultrafast population $(\sim 12 \mathrm{fs})$, followed by a slower depopulation ( $\sim 250 \mathrm{fs})$ of highly excited states of atomic fragments generated in the process of XFEL-induced nanoplasma formation. Inelastic scattering of Auger electrons and interatomic Coulombic decay are suggested as the mechanisms populating and depopulating, respectively, these excited states. The observed response occurs within the typical $\mathrm{x}$-ray pulse durations and affects $\mathrm{x}$-ray scattering, thus providing key information on the foundations of $\mathrm{x}$-ray imaging with XFELs.

DOI: 10.1103/PhysRevX.8.031034

\section{INTRODUCTION}

Ultrashort pulses from x-ray free-electron lasers (XFELs) $[1,2]$ are revolutionizing the field of ultrafast structural investigations, allowing determination of so far unknown

*ueda@tagen.tohoku.ac.jp

Published by the American Physical Society under the terms of the Creative Commons Attribution 4.0 International license. Further distribution of this work must maintain attribution to the author(s) and the published article's title, journal citation, and DOI.
Subject Areas: Atomic and Molecular Physics,

Plasma Physics structures of, e.g., transient species [3] and proteins [4,5], and to probe light-induced structural changes [6,7]. XFEL pulses are currently giving access to a new regime of $\mathrm{x}$-ray intensities, opening new research avenues in studying the interaction between intense $\mathrm{x}$ rays and various forms of matter [8-11]. Understanding the ultrafast reactions induced by XFEL pulses is of fundamental interest, as well as of crucial importance for structure determination $[12,13]$.

It is known that nanoplasma is formed whenever a nanosized species is irradiated by an intense infrared (IR) or extreme-ultraviolet (XUV) laser pulse [14-16]. Electrons are stripped away from the individual atoms by 
the laser, but trapped by the highly charged ionic species in the nanoplasma. The IR laser heats the plasma mainly via inverse bremsstrahlung and the XUV laser via photoionization $[15,16]$. Recently, nanoplasma formation was specifically reported for an atomic cluster irradiated by an XFEL pulse in the hard-X-ray regime [17]. In contrast to the former, nanoplasma formation by hard-x-ray pulses is highly indirect. Here, secondary electrons are generated either by inelastic scattering of the electrons ejected in the cascade of intra- and interatomic relaxation steps of the deep core holes initially created on individual atoms, or by the cascade itself, in its later stages. These low-energy electrons are trapped by the highly charged cluster forming the nanoplasma [17]. Also in the hard-x-ray regime, the nanoplasma formation is expected to occur for any nanometer-size matter irradiated by intense XFEL pulses. The evolution of the nanoplasma produced by XUV pulses was probed in the range of picoseconds [18]; on this timescale, follow-up electron-ion recombination processes occur during the expansion of the nanoplasma. In contrast, the nanoplasma formation process itself, which is expected to occur on a shorter time range, has never been observed to date. This is due to the lack of methods with sufficient temporal resolution. Here, we present the first femtosecond time-resolved observation of nanoplasma formation from an atomic cluster irradiated by an XFEL pulse, the birth of a nanoplasma.

\section{RESULTS AND DISCUSSIONS}

\section{A. Xe atoms}

We first show results for isolated Xe atoms. This is done, on one hand, to demonstrate the available temporal resolution after jitter corrections for the absolute time delay determination and, on the other hand, to emphasize the fundamental difference created by the presence of an environment in the clusters on the processes triggered by the absorption of an x-ray photon. As reported earlier [19], the irradiation of isolated Xe atoms with XFEL pulses produces a wide distribution of highly charged ions (up to $\mathrm{Xe}^{26+}$ ) with a maximum at $\mathrm{Xe}^{8+}$ and with a nearly negligible fraction of low-charged ions $\left(\mathrm{Xe}^{+}\right.$and $\left.\mathrm{Xe}^{2+}\right)$. The highly charged ions are produced by a sequential multiphoton multiple ionization and Auger cascades [19]. In the present study, the kinetic energies of the highly charged ions $\mathrm{Xe}^{m+}(m=3-21)$ were thermal $(\sim 0.04 \mathrm{eV}$ in the present experimental conditions), resulting in very sharp peaks of the individual isotopes of the ions in the time-of-flight spectra, as also seen in Ref. [19]. It evidences that these highly charged Xe ions were not generated via Coulomb explosion of highly charged Xe clusters, but via Auger cascades in the isolated Xe atoms. Irradiating in addition with a near-IR (NIR) pulse, we found an enhancement of the $\mathrm{Xe}^{13+}$ ion yield at a certain time delay with respect to the XFEL-pump pulse, as depicted in Fig. 1(a). Similar enhancements were also found for the yields of

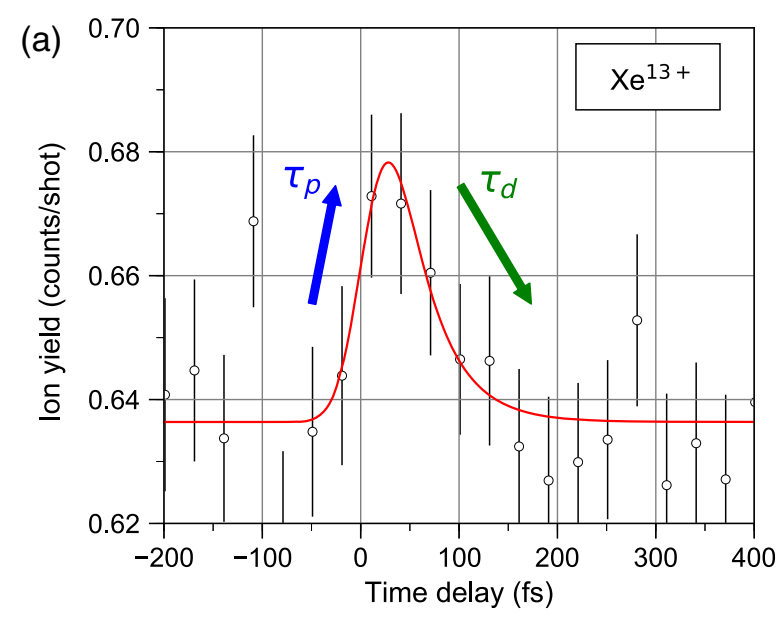

(b)
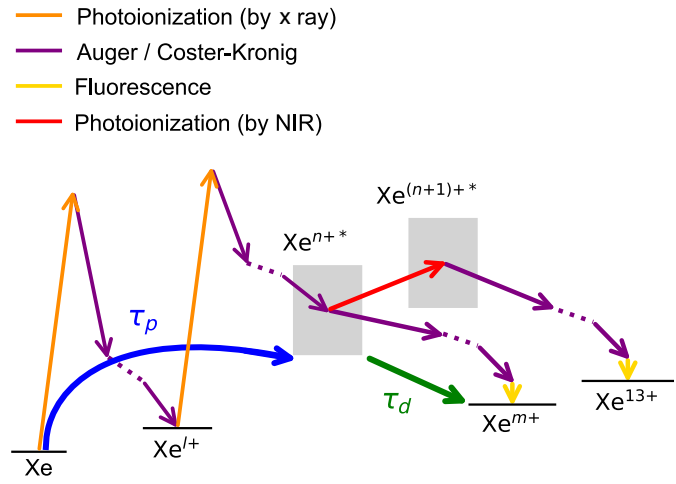

FIG. 1. Ionization of $\mathrm{Xe}^{n+}$ states by the NIR laser in isolated atoms. (a) $\mathrm{Xe}^{13+}$ yields as a function of time delay of the NIRprobe pulses $(800 \mathrm{~nm})$ with respect to the XFEL-pump pulses $(5.5 \mathrm{keV})$. The experimental results are given by white circles. The red solid curve shows the results of the fit with a product of two exponential functions with increasing and decreasing time constants of $\tau_{p}$ and $\tau_{d}$, respectively. The function was convoluted with a Gaussian function accounting for the instrumental function with a width $\sigma . \tau_{p} \simeq 10 \mathrm{fs}$ and $\tau_{d} \simeq 40 \mathrm{fs}$ are the time constants for the population and depopulation of $\mathrm{Xe}^{n+}(n \leq 12)$ states, respectively. The zero time delay was obtained by the fit with an accuracy of \pm 20 fs. The fitting parameter $\sigma \simeq 20$ fs (rms) or $\sim 50$ fs (FWHM) gives the time resolution. All data points in the time delay range from -800 to +1500 fs have been used for the fit. (b) Schematic diagram for the ionization of the $\mathrm{Xe}^{n+}(n \leq m)$ states which decay into $\mathrm{Xe}^{m+}(m<13)$ states without NIR probe. The orange, purple, yellow, and red arrows show the photoionization of the Xe atoms and Xe ions by x ray, Auger cascades of core-ionized Xe ions, fluorescence, and the photoionization of transient $\mathrm{Xe}^{n+}$ states by the NIR laser, respectively.

$\mathrm{Xe}^{11+}$ and $\mathrm{Xe}^{12+}$ with lower statistics. We note that due to saturation effects of the ion spectrometer within the present experimental conditions, the respective variations in the yields of higher and lower charge-state ions could not be observed (see Supplemental Material [20]).

In the present atomic measurement, the transient excited states that can be ionized by the NIR pulse are most probably highly excited states of $\mathrm{Xe}^{n+}$ with $n \leq 12$. These 
states are expected to be populated within $10 \mathrm{fs}[19,21]$ and depopulated via the subsequent Auger decay in a few tens of fs at most (see Ref. [19]). A schematic view of the NIRprobe process for the present measurement is given in Fig. 1(b). By fitting we determined that the accuracy of the extracted absolute time delay is $\pm 20 \mathrm{fs}$. The fitting details are given in the Appendix. The temporal width of the jittercorrected pump-probe delay measurement estimated from the fitted Gaussian width is $\sigma \simeq 20 \mathrm{fs}$ (rms) or $\sim 50 \mathrm{fs}$ (FWHM). The temporal width without shot-by-shot jitter corrections is $\sim 700 \mathrm{fs}$ (FWHM) and thus the jitter corrections lead to an improvement of the temporal resolution by more than an order of magnitude. The extracted decay-time constant $\tau_{d}$ is $\sim 40 \mathrm{fs}$.

\section{B. Xe clusters}

Let us now discuss the results of the Xe clusters. We found that the vast majority of fragment ions are $\mathrm{Xe}^{+}$and $\mathrm{Xe}^{2+}$. The kinetic energies of $\mathrm{Xe}^{+}$and $\mathrm{Xe}^{2+}$ ions were several tens of $\mathrm{eV}$, evidencing that these ions were produced by the cluster explosion, contrasting with the nonenergetic highly charged $\mathrm{Xe}^{m+}(m=3-21)$ ions produced from the uncondensed atoms present in the beam. A small amount of singly charged cluster ions $\mathrm{Xe}_{k}^{+}(k \geq 2)$ was also observed. The fact that no highly charged energetic ions coming from the cluster were observed illustrates that the charge accumulated on the initially ionized atoms during the decay cascade spreads over the cluster extremely quickly before the Coulomb explosion takes place. Previous studies on rare-gas clusters interacting with an XUV laser $[15,16,22-24]$ also show that the dominant ionic fragments from clusters are much lower charged ones than those from the isolated atoms, illustrating that the charge transfer occurs much faster than the cluster disintegration. The measured kinetic energy distributions of $\mathrm{Xe}^{+}$and $\mathrm{Xe}^{2+}$ are similar to those reported for the XUV laser irradiation (see, e.g., Ref. [24]). Namely, the kinetic energy of $\mathrm{Xe}^{+}$ distributes widely from a few $\mathrm{eV}$ to $\sim 100 \mathrm{eV}$, illustrating that $\mathrm{Xe}^{+}$ions are produced both in the core and at the periphery of the cluster. Kinetic energies of $\mathrm{Xe}^{2+}$ ions, on the other hand, distribute from a few tens of $\mathrm{eV}$ to $\sim 160 \mathrm{eV}$, with an average value of $\sim 80 \mathrm{eV}$, illustrating that they are generated at the periphery of the cluster.

A simple electrostatic estimate (see Supplemental Material [20]) based on the measured kinetic energy of $\mathrm{Xe}^{2+}$ gives the total charge of the cluster, or the number of the electrons emitted from the cluster, to be $\sim 120$. Assuming that the total charge of a cluster is distributed in such a way that the total Coulomb energy is minimized [24] would give a ratio of $\mathrm{Xe}^{+}$to $\mathrm{Xe}^{2+}$ of $\sim 2.5$, which is close to the present observations $(\sim 3)$. The photoionization cross section of neutral $\mathrm{Xe}$ atoms at $5.5 \mathrm{keV}$ is $\sim 0.166 \mathrm{Mb}$ [19]. Therefore, with the present peak fluence of the XFEL pulse $\left(\sim 30 \mu \mathrm{J} / \mu \mathrm{m}^{2}\right)$, the ionization probability of each Xe atom in the cluster is $\sim 57 \%$ and, thus, $\sim 2800$ atoms in the cluster of $\sim 5000$ atoms may be core ionized. We note, however, that the present experimental data have been averaged over the focal volume of the XFEL pulses [11,15], which means a larger contribution from lower XFEL intensities (see Supplemental Material [20] for a discussion on the dependence on the XFEL intensity). Therefore, the averaged total charge of the cluster discussed above is more than an order of magnitude smaller than that expected from the peak fluence, taking into account the very efficient three-body recombination in the core part of the dense nanoplasma [10].

Let us first focus on the $\mathrm{Xe}^{2+}$ yield. As seen in Fig. 2(a), a gradual increase of the $\mathrm{Xe}^{2+}$ yield with the time delay is
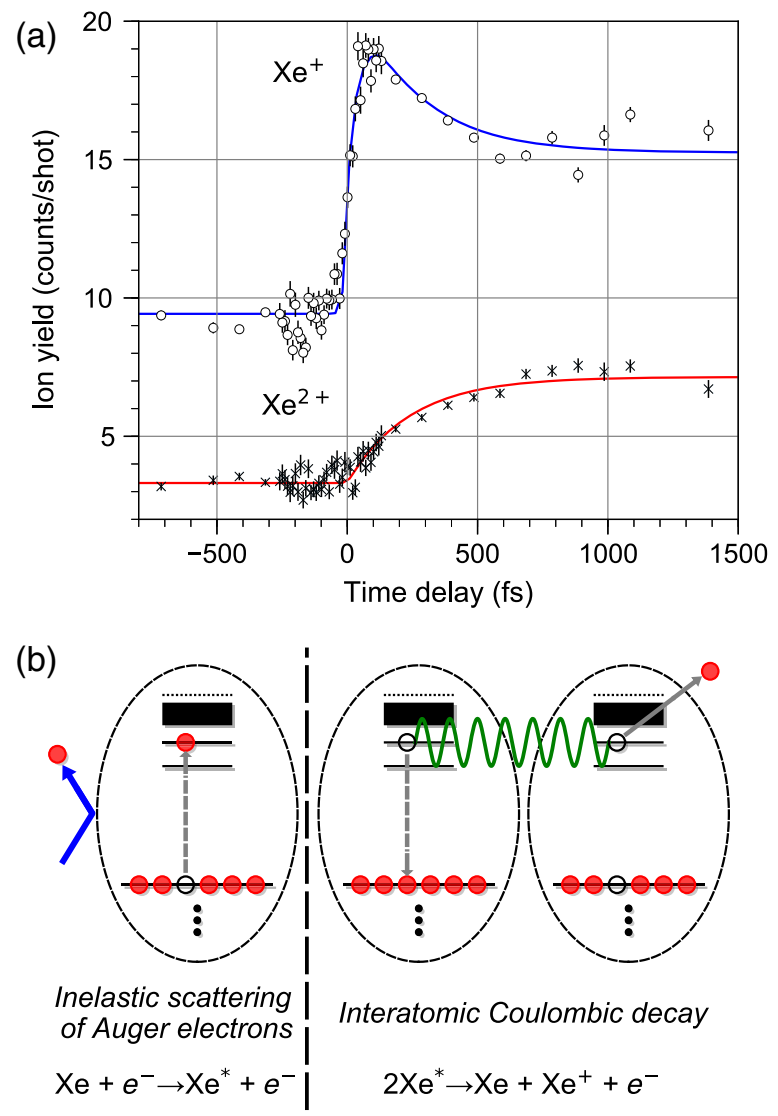

FIG. 2. Ultrafast dynamics of XFEL-induced nanoplasma. (a) $\mathrm{Xe}^{+}$and $\mathrm{Xe}^{2+}$ yields as a function of the time delay of the NIR-probe pulse $(800 \mathrm{~nm})$ with respect to the XFEL-pump pulse $(5.5 \mathrm{keV})$. The experimental $\mathrm{Xe}^{+}$and $\mathrm{Xe}^{2+}$ yields are given by circles and crosses, respectively. The solid curves show results of the theoretical modeling of the process of NIR laser heating up of XFEL-induced nanoplasma, leading to the gradual increase of the $\mathrm{Xe}^{+}$and $\mathrm{Xe}^{2+}$ yields, as well as the process of formation of excited atoms $\mathrm{Xe}^{*}$ that can pairwise undergo interatomic Coulombic decay (ICD), or alternatively be directly ionized by the delayed NIR-probe pulse, resulting in a sharp jump and a slower depletion in the $\mathrm{Xe}^{+}$yield. The highly excited states are formed by the inelastic scattering of the Auger electrons during the early stages of nanoplasma formation. (b) Schematic diagrams for the inelastic scattering of Auger electrons and the ICD. 
observed. This increase can be explained as follows. After a certain number of ionization events via photoemission and the first few Auger decay steps, the cluster is charged to such a degree that its Coulomb potential becomes deep enough to trap the relatively low-energetic electrons that are ejected in the later stages of the decay cascade or generated by electron-impact ionization (secondary electrons). These trapped, quasifree electrons, together with (as indicated by experiment) low-charge ions, form the nanoplasma [17]. The nanoplasma electrons may then acquire kinetic energy from the additional NIR laser field and further ionize neutral Xe to $\mathrm{Xe}^{+}$and $\mathrm{Xe}^{+}$to $\mathrm{Xe}^{2+}$ by collisional ionization processes. This clearly enhances the $\mathrm{Xe}^{+}$and $\mathrm{Xe}^{2+}$ yields, but why is the latter growing with the delay time as seen in Fig. 2(a)? When nanoplasma is formed by the XFEL pulse during the first few femtoseconds, the quasifree electrons are so dense that they shield the electric field inside the nanoplasma, making it much lower than the field outside of the cluster [14]. The electron density, however, decreases with time due to the nanoplasma expansion $[23,25]$. Thermal electron emission [17], as well as electron-ion recombination within the nanoplasma $[10,18,25]$, may also contribute to the loss of quasifree electrons. With the decrease of the electron density, the nanoplasma starts to absorb NIR light more efficiently via the surface plasmon resonance effect $[14,16]$ (see Supplemental Material [20]). As a result, the $\mathrm{Xe}^{2+}$ yield gradually increases as a function of the time delay, as seen in Fig. 2(a).

In contrast to the gradual increase of the $\mathrm{Xe}^{2+}$ yield, the $\mathrm{Xe}^{+}$yield exhibits a highly unexpected abrupt jump at zero time delay, followed by a slower decrease as a function of the time delay, as seen in Fig. 2(a). This behavior indicates that, besides the NIR heating up during the gradual expansion of the nanoplasma discussed above, the NIR pulse probes another, much faster process: the transient population by the XFEL pulse of highly excited states of neutral atomic fragments that can be ionized by the NIR pulse. This is reminiscent of the case of the $\mathrm{Xe}^{13+}$ yield in the isolated atoms discussed above.

How are the excited neutral atomic states populated so fast, and what is the mechanism by which they later generate $\mathrm{Xe}^{+}$? Such a short time indicates that the excitations are created during the nanoplasma formation and not afterwards, when they can be produced by, for example, three-body recombination of quasifree plasma electrons $[10,18]$. The most probable mechanism is inelastic scattering of the Auger electrons emitted during the initial cascade from neutral atoms in the cluster, as schematically depicted in Fig. 2(b). The population by the XFEL pulse of the neutral excited states is expected to deplete by a variant of the interatomic Coulombic decay (ICD) [26] in which two excited atoms exchange energy ionizing one of them and deexciting the other [27-30] [see Fig. 2(b)]. As many highly excited xenon atoms are created in the cluster, a cascade of such ICD processes is expected [30].
To check this mechanism and to extract some relevant characteristics of the processes involved, we performed model calculations based on a system of rate equations. The equations describe evolutions of the amount of $\mathrm{Xe}, \mathrm{Xe}^{*}$, $\mathrm{Xe}^{+}$, and $\mathrm{Xe}^{2+}$ exposed to two Gaussian laser pulses: a 10-fs XFEL-pump pulse and a 32-fs NIR-probe pulse. The pump pulse can produce $\mathrm{Xe}^{*}, \mathrm{Xe}^{+}$, and $\mathrm{Xe}^{2+}$ with probabilities (cross sections) taken as free parameters to be determined by the fitting to the experimental data. The probe pulse can ionize $\mathrm{Xe}^{*}$ with a constant probability, as well as $\mathrm{Xe}$ to $\mathrm{Xe}^{+}$and $\mathrm{Xe}^{+}$to $\mathrm{Xe}^{2+}$ with probabilities which depend on the time of arrival of the probe pulse with respect to the XFEL pulse. The latter simulates the increase of NIR absorption cross section with the cluster expansion through the surface-plasmon-resonance mechanism discussed above. The functional dependence of these probabilities is taken to be $A \theta\left(t-\tau_{0}\right)\left\{1-\exp \left[-\left(t-\tau_{0}\right) / \tau\right]\right\}$, where $\theta\left(t-\tau_{0}\right)$ is the Heaviside step function and $\tau_{0}$ is the time of arrival of the XFEL-pump pulse. In addition, a fraction of $\mathrm{Xe}^{*}$ atoms can decay pairwise by ICD to $\mathrm{Xe}^{+}$ and $\mathrm{Xe}$. The resulting set of rate equations, containing 10 free parameters, has been solved numerically by scanning the pump-probe time delay from -750 to $1500 \mathrm{fs}$, optimizing the parameters such that we obtain the best fit to the experimental data for $\mathrm{Xe}^{1+}$ and $\mathrm{Xe}^{2+}$ yields (see the Appendix). The resulting $\mathrm{Xe}^{+}$and $\mathrm{Xe}^{2+}$ yields are shown in Fig. 2(a).

The extracted optimum parameters show that the population of the excited xenon atoms should occur within $\sim 12$ fs, which corroborates well with the time constant ( $\sim 10 \mathrm{fs}$ ) found for the formation of $\mathrm{Xe}^{13+}$ in the isolated atoms [see Figs. 1(a) and (b)], indicating that the excited Xe* are formed during the initial Auger cascade. The optimum value for the ICD decay time is obtained to be $250 \mathrm{fs}$, yet with a rather large confidence interval, meaning that this value can be taken only as indicative for the timescale of the process. The time constant of the ICD process depends on the excited states involved, on the distance between the excited atoms, and on their number, being thus rather difficult to estimate from first principles for the present conditions. However, a number of theoretical and experimental studies have shown [27-32] that the timescale of ICD in rare-gas clusters is typically a few hundred femtoseconds. Therefore, the present extracted averaged time constant of $\sim 250$ fs is fully within the range of timescales to be expected for ICD mechanisms in such systems.

The previous study by Tachibana et al. [17] in the hard$\mathrm{x}$-ray regime revealed that a nanoplasma is efficiently formed from a cluster by the trapping of low-energy electrons. In the present study, we found the abrupt increase of $\mathrm{Xe}^{+}$yield due to the ionization of the highly excited atoms with the NIR pulse. Ultrafast production of these highly excited atoms is attributed to the inelastic scattering of the electrons ejected in the cascade of intra- and interatomic relaxation steps of a deep core hole initially 
created on individual atoms, at the very beginning of, or at the moment of, the nanoplasma formation. The highly excited states which are populated in $\sim 10$ fs and depopulated in $\sim 250 \mathrm{fs}$, in many atomic species inside the cluster, can be regarded as the gateway states towards nanoplasma formation. We succeeded to probe these gateway states driving the birth of the nanoplasma.

In the end, we note that the XFEL-intensity dependence of $\mathrm{Xe}^{+}$and $\mathrm{Xe}^{2+}$ yields (see Supplemental Material [20]) seems to indicate that the average ICD time increases with the increase of the XFEL intensity. This can be explained as follows. The number of directly created $\mathrm{Xe}^{+}\left(\right.$and $\mathrm{Xe}^{2+}$ ) ions increases with the intensity of the XFEL pulse. The higher charge accumulated hinders, and may even energetically close, the very efficient close-to-threshold intraRydberg ICD transitions between highly excited atoms [30], leading in that way to an increase in the average ICD time constant.

\section{CONCLUSION}

In conclusion, the dramatically improved time resolution of an XFEL-pump-NIR-probe experiment in the hard-Xray regime allowed us to monitor the "birth" of a nanoplasma by capturing the ultrafast population ( $\sim 12 \mathrm{fs})$ and depopulation ( $250 \mathrm{fs})$ of excited states of cluster atoms during nanoplasma formation in Xe clusters. The measured time constants may be understood by the inelastic scattering of electrons produced in the Auger cascade followed by (a cascade of) interatomic decay processes such as ICD. The present experiments allow us for the first time to gain a fundamental insight into the ultrafast reactions induced by XFEL pulses, which should also be of crucial relevance for the use of XFEL pulses to determine the structure of nanosized objects [12,13]. The present femtosecond timeresolved method is versatile and can be widely applied to study electron and nuclear dynamics induced by the interaction of hard-XFEL pulses with any form of matter.

\section{ACKNOWLEDGMENTS}

We are grateful to the late Makoto Yao for his invaluable contributions to the present work. The experiments were performed at SPring-8 Angstrom Compact free electron LAser (SACLA) with the approval of JASRI and the program review committee (No. 2015B8057). This study was supported by the X-ray Free Electron Laser Utilization Research Project and the X-ray Free Electron Laser Priority Strategy Program of the Ministry of Education, Culture, Sports, Science and Technology of Japan (MEXT), by the Proposal Program of SACLA Experimental Instruments of RIKEN, by the Japan Society for the Promotion of Science (JSPS) KAKENHI Grants No. JP21244042, No. JP23241033, No. JP15K17487, and No. JP16K05016, and by the Institute of Multidisciplinary
Research for Advanced Materials (IMRAM) project. H. F. and K. U. acknowledge support by the Dynamic Alliance for Open Innovation Bridging Human, Environment and Materials. D. I. and Y. I. acknowledge support by IMRAM, Tohoku University. K. N. and S. W. acknowledge support by the Research Program of "Dynamic Alliance for Open Innovation Bridging Human, Environment and Materials" in "Network Joint Research Center for Materials and Devices". T. Takanashi acknowledges support by JSPS KAKENHI Grant No. JP16J002270. D. Y. acknowledges support by the Grant-in-Aid of Tohoku University Institute for Promoting Graduate Degree Programs Division for Interdisciplinary Advanced Research and Education. T. N. acknowledges support by the Research Program for Next Generation Young Scientists of "Dynamic Alliance for Open Innovation Bridging Human, Environment and Materials" in "Network Joint Research Center for Materials and Devices". A. I. K. thanks DFG for financial support. M. S. and G. K. gratefully acknowledge financial support by BMBF (Grant No. 05K16RF3) and the DFG Research Unit 1789 Grant No. 203306641. Y. K. was partially supported by the U.S. Department of Energy, Office of Science, Basic Energy Sciences, Chemical Sciences, Geosciences, and Biosciences Division. K. Kooser acknowledges the financial support provided by the Estonian Research Council (Grant No. PUT735) and from the Väisälä Foundation of the Finnish Academy of Science and Letters.

\section{APPENDIX: MATERIALS AND METHODS}

\section{Experimental apparatus}

Figure 3 shows a schematic picture of the XFEL-pumpNIR-probe setup used in the present measurements. The experiments were performed at the experimental hutch 4 (EH4) of beam line 3 (BL3) [33] of the XFEL facility in Japan, SACLA [2]. SACLA generated $5.5-\mathrm{keV}$ x-ray pulses with a bandwidth of $\sim 40 \mathrm{eV}$ (FWHM) and a repetition rate of $30 \mathrm{~Hz}$. The XFEL peak fluence in the interaction point was measured to be $30 \mu \mathrm{J} / \mu \mathrm{m}^{2}$ via an established calibration procedure using argon atoms [19]. The Xe clusters with average size of $\langle N\rangle=5000$ atoms were prepared by adiabatic expansion of atomic Xe gas. Uncondensed Xe atoms were also prepared by setting the jet parameters such that isolated Xe atoms remain in the cluster beam. In both cases (clusters and uncondensed atoms), when the time delay was positive, the samples were first irradiated by XFEL pulses, and further by the NIRprobe laser with a wavelength of $800 \mathrm{~nm}$. The pulse duration of the NIR laser was measured to be $32 \mathrm{fs}$ (FWHM) and the peak fluence was $15 \mathrm{~nJ} / \mu \mathrm{m}^{2}$ $\left(4.8 \times 10^{13} \mathrm{~W} / \mathrm{cm}^{2}\right.$ peak intensity). The charge-resolved $\mathrm{Xe}$ ion yields were measured as a function of the time delay of the NIR-probe pulse relative to the XFEL-pump pulse, 


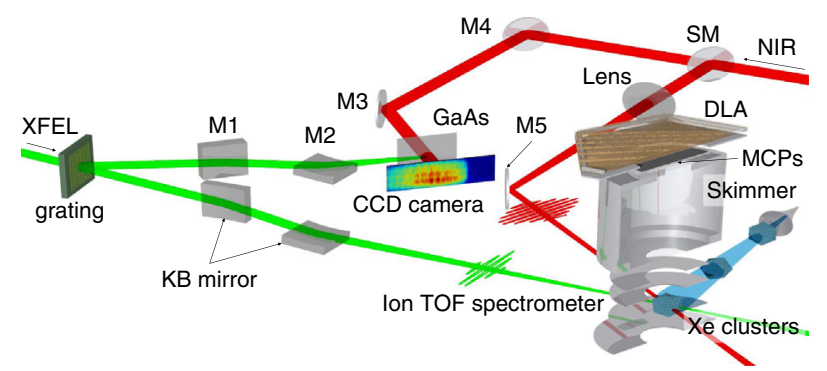

FIG. 3. Schematic picture of the XFEL-pump-NIR-probe experiment. The incident XFEL pulse impinges the grating [34], which generates multiple beams. A main part of the XFEL beam is focused on the interaction point by Kirkpatrick-Baez (KB) mirrors. The XFEL pulse $[5.5 \mathrm{keV},<10 \mathrm{fs}$ (FWHM), $30 \mu \mathrm{J} / \mu \mathrm{m}^{2}$ ] and the NIR laser pulse [800 nm, $32 \mathrm{fs}$ (FWHM), $\left.15 \mathrm{~nJ} / \mu \mathrm{m}^{2}\right]$ illuminate the Xe clusters $(\langle N\rangle=5000$ atoms $)$ directed to the interaction point through a skimmer. The ion time-of-flight (TOF) spectrometer accelerates the produced ions, and the position sensitive detector, constructed with microchannel plates (MCPs) and a delay-line anode (DLA), detects them. The different ion charges are clearly separated in the time-offlight spectra. The other parts of the XFEL and NIR laser illuminate single crystals from gallium arsenide (GaAs) serving as targets for the arrival timing monitor [34]. The CCD camera records the spatial modulation of the optical transmittance of the NIR laser in order to determine the accurate time delay of the NIR-probe pulse with respect to the XFEL-pump pulse. M1 and M2 are mirrors for the XFEL beam, M3-M5 are mirrors for the NIR laser, and SM is a split mirror for the NIR laser.

with an ion time-of-flight spectrometer [19]. The heart of the experiment is how to determine the delay of the NIRprobe pulse relative to the XFEL-pump pulse at a resolution of tens of femtoseconds, overcoming temporal jitters of $\sim 1$ ps. For this purpose, a portion of the XFEL and NIR pulses were separated by a grating and a split mirror, respectively, and were injected into a gallium arsenide crystal used as a target for the arrival timing monitor [34]. The temporal jitters between arrival times of the XFEL and NIR pulses were measured on a shot-by-shot basis.

\section{Analysis}

To analyze the temporal behavior of the measured ion yields, we refer to the XUV-pump-UV-probe measurements on Xe atoms at FLASH in Germany, where an enhancement for the $\mathrm{Xe}^{4+}$ yield was observed as a function of the time delay. The enhancement was due to the ionization of the transient $\mathrm{Xe}^{2+}\left(5 p^{-4} n \ln ^{\prime} l^{\prime}\right)$ states to $\mathrm{Xe}^{4+}\left(5 p^{-4}\right)$ by the UV-probe pulse, before they autoionize to $\mathrm{Xe}^{3+}\left(5 p^{-3}\right)$ [35]. The temporal dependence of the population of the transient states was described by an increasing (populating) and decreasing (depopulating) double exponential function with the exponential time constants $\tau_{p}$ and $\tau_{d}$, respectively:

$$
f(t)=\left\{\begin{array}{cc}
0 & \left(t<t_{0}\right) \\
a\left[1-\exp \left(-\frac{t-t_{0}}{\tau_{p}}\right)\right] \exp \left(-\frac{t-t_{0}}{\tau_{d}}\right) & \left(t \geq t_{0}\right)
\end{array}\right.
$$

where $t_{0}$ was the zero time delay.

We employ the above formula to describe the temporal dependence of the population of the transient states in $\mathrm{Xe}^{n+}$ $(n \leq 12)$. We also take into account the instrumental function. Then, the fitting function $F(t)$ can be described as a convolution of the above double exponential function $f(t)$ and a Gaussian function $g(t)$ :

$$
\begin{aligned}
& F(t)=(f * g)(t)+C, \\
& g(t)=\frac{1}{\sqrt{2 \pi} \sigma} \exp \left(-\frac{t^{2}}{2 \sigma^{2}}\right),
\end{aligned}
$$

where $\sigma$ is the width of the Gaussian function and $C$ is a time-independent constant.

In order to fit the $\mathrm{Xe}^{13+}$ curve in Fig. 1(a), the time constant of the exponential increase $\tau_{p}$ was assumed to be $10 \mathrm{fs}$. Under this assumption we obtained the uncertainty of the absolute time delay of $\pm 20 \mathrm{fs}$, the temporal width $\sigma \simeq 20 \pm 20 \mathrm{fs}$ (rms), and the decay-time constant $\tau_{d} \simeq 40 \pm 40 \mathrm{fs}$. The large uncertainties stem from the strong correlations between these parameters and the poor statistics. Adopting the Gaussian width to be given by the convolved width of $35 \mathrm{fs}$ of the XFEL (10 fs) and NIR (32 fs) pulses, we obtain the decay-time constant $\tau_{d}=43 \pm 31 \mathrm{fs}$. When we scan $\tau_{p}$ from 3 to $30 \mathrm{fs}, t_{0}$ varies by $10 \mathrm{fs}, \sigma$ by $2 \mathrm{fs}$, and $\tau_{d}$ by 2 fs. To evaluate absolute uncertainties, these small variations may need to be added to the statistical uncertainties described above.

The evolution of $\mathrm{Xe}^{+}$and $\mathrm{Xe}^{2+}$ yields as a function of the XFEL-NIR delay have been modeled by a set of equations, describing the rate with which the amounts of $\mathrm{Xe}, \mathrm{Xe}^{*}, \mathrm{Xe}^{+}$, and $\mathrm{Xe}^{2+}$ are changing by transitions induced by the pump and the probe pulses, and by the ICD process, which produces a neutral and a singly ionized xenon from two excited ones.

The action of the laser pulses has been modeled as follows. We assume that the pulse induces a smooth quantum transition between an initial and a final state (linear regime); i.e., the population transfer follows the smooth function $\xi(t)$. An appropriate choice for such a transition function is

$$
\xi(t)=\frac{1}{1+\exp (-\alpha t)},
$$

which goes smoothly from 0 to 1 and the parameter $\alpha$ determines the duration of the transition. The rate of the population transfer $R(t)$ is given by the derivative of the function $\xi(t)$ multiplied by the strength of the transition $\kappa$ : 


$$
R(t)=\kappa \frac{\alpha}{2+2 \cosh (\alpha t)} .
$$

It was shown [36] that in a two-level system a population transfer following an arbitrary smooth function $\xi(t)$ can be performed with a resonant pulse with an envelope given by

$$
E(t)=\frac{\dot{\xi}(t)}{\sqrt{\xi(t)[1-\xi(t)]}},
$$

where $\dot{\xi}(t)$ denotes time derivative of $\xi(t)$. This expression can be used to relate the FWHM, or the duration of the later pulse, with the duration of the transition $\alpha$. Using Eq. (A3) for the function $\xi(t)$ one can easily get

$$
\mathrm{FWHM}=\frac{2}{\alpha} \operatorname{arctanh}\left(\frac{4 \sqrt{3}}{7}\right) .
$$

For simulating the increasing with the cluster expansion probability to absorb NIR light (through the surfaceplasmon-resonance mechanism), the strength of the transition $\kappa$ is taken to be time dependent with the following form,

$$
\kappa(t)=A \theta\left(t-\tau_{0}\right)\left[1-\exp \left(-\frac{t-\tau_{0}}{\tau}\right)\right],
$$

where $\tau_{0}$ is the time of arrival of the XFEL-pump pulse.

Finally, the ICD mechanism converts two excited xenons into a neutral and a singly ionized one with a rate $\kappa_{\mathrm{ICD}}$. For accounting that not all excited xenon atoms will decay by $\mathrm{ICD}$, in the rate equations we assume that the pump pulse produces two "types" Xe*: ICD active and ICD inactive. Both of them can be ionized by the NIR pulse with the same rate.

The resulting set of rate equations thus contains 10 parameters: the rates with which the pump XFEL pulse produces $\mathrm{Xe}^{*}$ (ICD active and ICD inactive), $\mathrm{Xe}^{+}$and $\mathrm{Xe}^{2+}$ from neutral xenon, the rates with which the probe NIR pulse produces $\mathrm{Xe}^{+}$from $\mathrm{Xe}^{*}, \mathrm{Xe}^{+}$from $\mathrm{Xe}^{*}$, and $\mathrm{Xe}^{2+}$ from $\mathrm{Xe}^{+}$, and the ICD rate. These equations have been solved numerically, scanning the delay between the XFEL and NIR pulses from -750 to 1500 fs and optimizing the parameters such that we obtain the best least-squares fit to the experimental data for the $\mathrm{Xe}^{+}$and $\mathrm{Xe}^{2+}$ yields. The Rosenbrock evolutionary algorithm for nonlinear leastsquares optimization as implemented in the DAKOTA package [37] has been used by imposing a set of constraints on the parameters extracted from physical considerations and classical estimations for the cluster expansion speed (see Supplemental Material [20]).

[1] P. Emma, R. Akre, J. Arthur, R. Bionta, C. Bostedt et al., First Lasing and Operation of an Angstrom-Wavelength Free-Electron Laser, Nat. Photonics 4, 641 (2010).
[2] T. Ishikawa, H. Aoyagi, T. Asaka, Y. Asano, N. Azumi et al., A Compact X-Ray Free-Electron Laser Emitting in the Sub-Ångström Region, Nat. Photonics 6, 540 (2012).

[3] N. D. Loh, C. Y. Hampton, A. V. Martin, D. Starodub, R. G. Sierra et al., Fractal Morphology, Imaging and Mass Spectrometry of Single Aerosol Particles in Flight, Nature (London) 486, 513 (2012).

[4] L. Redecke, K. Nass, D. P. DePonte, T. A. White, D. Rehders et al., Natively Inhibited Trypanosoma brucei Cathepsin B Structure Determined by Using an X-Ray Laser, Science 339, 227 (2013).

[5] M. Suga, F. Akita, K. Hirata, G. Ueno, H. Murakami et al., Native Structure of Photosystem II at $1.95 \AA$ Resolution Viewed by Femtosecond X-Ray Pulses, Nature (London) 517, 99 (2015).

[6] R. Mankowsky, A. Subedi, M. Först, S. O. Mariager, M. Chollet et al., Nonlinear Lattice Dynamics as a Basis for Enhanced Superconductivity in $\mathrm{YBa}_{2} \mathrm{Cu}_{3} \mathrm{O}_{6.5}$, Nature (London) 516, 71 (2014).

[7] K. H. Kim, J. G. Kim, S. Nozawa, T. Sato, K. Y. Oang et al., Direct Observation of Bond Formation in Solution with Femtosecond X-Ray Scattering., Nature (London) 518, 385 (2015).

[8] H. Yoneda, Y. Inubushi, K. Nagamine, Y. Michine, H. Ohashi et al., Atomic Inner-Shell Laser at 1.5-Ångström Wavelength Pumped by an X-Ray Free-Electron Laser, Nature (London) 524, 446 (2015).

[9] L. Young, E. P. Kanter, B. Krässig, Y. Li, A. M. March et al., Femtosecond Electronic Response of Atoms to UltraIntense X-Rays, Nature (London) 466, 56 (2010).

[10] H. Thomas, A. Helal, K. Hoffmann, N. Kandadai, J. Keto et al., Explosions of Xenon Clusters in Ultraintense Femtosecond X-Ray Pulses from the LCLS Free Electron Laser, Phys. Rev. Lett. 108, 133401 (2012).

[11] T. Gorkhover, M. Adolph, D. Rupp, S. Schorb, S. W. Epp et al., Nanoplasma Dynamics of Single Large Xenon Clusters Irradiated with Superintense X-Ray Pulses from the Linac Coherent Light Source Free-Electron Laser, Phys. Rev. Lett. 108, 245005 (2012).

[12] H. M. Quiney and K. A. Nugent, Biomolecular Imaging and Electronic Damage Using X-Ray Free-Electron Lasers, Nat. Phys. 7, 142 (2011).

[13] B. Ziaja, H. N. Chapman, R. Fäustlin, S. Hau-Riege, Z. Jurek, A. V. Martin, S. Toleikis, F. Wang, E. Weckert, and R. Santra, Limitations of Coherent Diffractive Imaging of Single Objects due to Their Damage by Intense X-Ray Radiation, New J. Phys. 14, 115015 (2012).

[14] T. Ditmire, T. Donnelly, A. M. Rubenchik, R. W. Falcone, and M. D. Perry, Interaction of Intense Laser Pulses with Atomic Clusters, Phys. Rev. A 53, 3379 (1996).

[15] U. Saalmann, C. Siedschlag, and J. M. Rost, Mechanisms of Cluster Ionization in Strong Laser Pulses, J. Phys. B 39, R39 (2006).

[16] T. Fennel, K.-H. Meiwes-Broer, J. Tiggesbäumker, P.-G. Reinhard, P. M. Dinh, and E. Suraud, Laser-Driven Nonlinear Cluster Dynamics, Rev. Mod. Phys. 82, 1793 (2010).

[17] T. Tachibana, Z. Jurek, H. Fukuzawa, K. Motomura, K. Nagaya et al., Nanoplasma Formation by High Intensity Hard X-Rays, Sci. Rep. 5, 10977 (2015). 
[18] B. Schütte, M. Arbeiter, T. Fennel, M. J. J. Vrakking, and A. Rouzée, Rare-Gas Clusters in Intense Extreme-Ultraviolet Pulses from a High-Order Harmonic Source, Phys. Rev. Lett. 112, 073003 (2014).

[19] H. Fukuzawa, S.-K. Son, K. Motomura, S. Mondal, K. Nagaya et al., Deep Inner-Shell Multiphoton Ionization by Intense X-Ray Free-Electron Laser Pulses, Phys. Rev. Lett. 110, 173005 (2013).

[20] See Supplemental Material at http://link.aps.org/ supplemental/10.1103/PhysRevX.8.031034 for the discussions of the counting efficiency loss for the highly charged ions, the total charge of the cluster, the surface plasmon resonance effects, the nanoplasma expansion, and the XFEL intensity dependence.

[21] K. Motomura, E. Kukk, H. Fukuzawa, S. ichi Wada, K. Nagaya et al., Charge and Nuclear Dynamics Induced by Deep Inner-Shell Multiphoton Ionization of $\mathrm{CH}_{3} \mathrm{I}$ Molecules by Intense X-Ray Free-Electron Laser Pulses, J. Phys. Chem. Lett. 6, 2944 (2015).

[22] M. Hoener, C. Bostedt, H. Thomas, L. Landt, E. Eremina, H. Wabnitz, T. Laarmann, R. Treusch, A. R. B. de Castro, and T. Möller, Charge Recombination in Soft X-Ray Laser Produced Nanoplasmas, J. Phys. B 41, 181001 (2008).

[23] H. Thomas, C. Bostedt, M. Hoener, E. Eremina, H. Wabnitz, T. Laarmann, E. Plönjes, R. Treusch, A. R. B. de Castro, and T. Möller, Shell Explosion and Core Expansion of Xenon Clusters Irradiated with Intense Femtosecond Soft X-Ray Pulses, J. Phys. B 42, 134018 (2009).

[24] H. Iwayama, A. Sugishima, K. Nagaya, M. Yao, H. Fukuzawa et al., Inhomogeneous Charge Redistribution in Xe Clusters Exposed to an Intense Extreme Ultraviolet Free Electron Laser, J. Phys. B 43, 161001 (2010).

[25] S. Vikrant and Z. Beata, Hydrodynamic Model for Expansion and Collisional Relaxation of X-Ray Laser-Excited Multi-Component Nanoplasma, Phys. Plasmas 23, 012710 (2016).

[26] L.S. Cederbaum, J. Zobeley, and F. Tarantelli, Giant Intermolecular Decay and Fragmentation of Clusters, Phys. Rev. Lett. 79, 4778 (1997).

[27] A. I. Kuleff, K. Gokhberg, S. Kopelke, and L. S. Cederbaum, Ultrafast Interatomic Electronic Decay in Multiply Excited Clusters, Phys. Rev. Lett. 105, 043004 (2010).
[28] P. V. Demekhin, K. Gokhberg, G. Jabbari, S. Kopelke, A. I. Kuleff, and L.S. Cederbaum, Overcoming Blockade in Producing Doubly Excited Dimers by a Single Intense Pulse and Their Decay, J. Phys. B 46, 021001 (2013).

[29] B. Schütte, M. Arbeiter, T. Fennel, G. Jabbari, A. I. Kuleff, M. J. J. Vrakking, and A. Rouzee, Observation of Correlated Electronic Decay in Expanding Clusters Triggered by Near-Infrared Fields, Nat. Commun. 6, 8596 (2015).

[30] K. Nagaya, D. Iablonskyi, N. V. Golubev, K. Matsunami, H. Fukuzawa et al., Interatomic Coulombic Decay Cascades in Multiply Excited Neon Clusters, Nat. Commun. 7, 13477 (2016).

[31] D. Iablonskyi, K. Nagaya, H. Fukuzawa, K. Motomura, Y. Kumagai et al., Slow Interatomic Coulombic Decay of Multiply Excited Neon Clusters, Phys. Rev. Lett. 117, 276806 (2016).

[32] T. Takanashi, N. V. Golubev, C. Callegari, H. Fukuzawa, K. Motomura et al., Time-Resolved Measurement of Interatomic Coulombic Decay Induced by Two-Photon Double Excitation of $\mathrm{Ne}_{2}$, Phys. Rev. Lett. 118, 033202 (2017).

[33] K. Tono, T. Togashi, Y. Inubushi, T. Sato, T. Katayama et al., Beamline, Experimental Stations and Photon Beam Diagnostics for the Hard X-Ray Free Electron Laser of SACLA, New J. Phys. 15, 083035 (2013).

[34] T. Katayama, S. Owada, T. Togashi, K. Ogawa, P. Karvinen et al., A Beam Branching Method for Timing and Spectral Characterization of Hard X-Ray Free-Electron Lasers, Struct. Dyn. 3, 034301 (2016).

[35] M. Krikunova, T. Maltezopoulos, A. Azima, M. Schlie, U. Frühling et al., Time-Resolved Ion Spectrometry on Xenon with the Jitter-Compensated Soft X-Ray Pulses of a FreeElectron Laser, New J. Phys. 11, 123019 (2009).

[36] N. V. Golubev and A. I. Kuleff, Control of Populations of Two-Level Systems by a Single Resonant Laser Pulse, Phys. Rev. A 90, 035401 (2014).

[37] B. M. Adams, L. E. Bauman, W. J. Bohnhoff, K. R. Dalbey, M. S. Ebeida et al., "DAKOTA, A Multilevel Parallel ObjectOriented Framework for Design Optimization, Parameter Estimation, Uncertainty Quantification, and Sensitivity Analysis: Version 6.0 User's Manual," Sandia National Laboratories, Technical Report No. SAND2014-4633, 2014. 